\title{
ANÁLISE MACROSCÓPICA DOS EFEITOS DOS HIALURONATOS E DO CORTICOSTEROIDE NO TRATAMENTO DA OSTEOARTROSE INDUZIDA EM JOELHOS DE COELHO
}

\author{
MACROSCOPIC ANALYSES OF THE EFFECTS OF HYALURONATES \\ AND CORTICOSTEROIDS ON INDUCED OSTEOARTHRITIS IN RABBITS' KNEES
}

\begin{abstract}
Mauro Batista Albano', Leandro Vidigal'2, Marcello Zaia de Oliveira ${ }^{3}$, Mario Massatomo Namba ${ }^{4}$, João Luiz Vieira da Silva ${ }^{5}$, Francisco de Assis Pereira Filho ${ }^{6}$, Marcio Alves Barbosa ${ }^{7}$, Elias Marcelo Batista da Silva ${ }^{8}$
\end{abstract}

\section{RESUMO}

Objetivo: Avaliar o efeito das injeções intra-articulares de corticosteroide e dos ácidos: hialurônico nativo e com cadeias ramificadas, na osteoartrose induzida experimentalmente. Métodos: 44 coelhos submetidos à ressecção do ligamento cruzado anterior foram divididos em quatro grupos de 11. Grupo 1: uma injeção intra-articular com solução salina por semana, durante 3 semanas. Grupo 2: três injeções, com intervalo semanal, de ácido hialurônico nativo. Grupo 3: três injeções, com intervalo semanal, de ácido hialurônico ramificado. Grupo 4: duas injeções de betametasona com intervalo de três semanas. Após 12 semanas da cirurgia a cartilagem articular dos planaltos tibiais foi avaliada por macroscopia. As alterações na superfície articular foram graduadas da seguinte maneira: grau 0 - superfície articular lisa, sem alterações de relevo; grau 1 - superfície rugosa, sem depressões; grau 2 - semelhante ao grau 1 porém com depressões na superfície; grau 3 - exposição do osso subcondral. A análise estatística consistiu na utilização do teste $t$ de Student, teste do Qui-quadrado e da análise de variância (Anova). O nível de significância utilizado foi de 5\%. Resultados: Observou-se haver diferença estatística entre os grupos 2, 3 e 4 e o grupo controle no desenvolvimento e gravidade da artrose, porém não houve diferença entre os grupos das drogas estudadas. Conclusão: Constatou-se atenuação da evolução do processo de osteoartrose, na mesma magnitude, com a utilização de injeções intra-articulares dos glicosaminoglicanos de baixo peso molecular e de alto peso molecular, assim como do corticosteroide betametasona quando comparados com placebo em joelhos de coelhos.

Descritores - Ácido hialurônico; Ligamento cruzado anterior; Osteoartrose; Joelho; Coelhos

\section{ABSTRACT}

Objective: The aim of this study was to evaluate the effects of intraarticular injections of corticosteroids, native hyaluronic acid and branched-chain hyaluronic acid in experimentally-induced osteoarthrosis. Methods: 44 rabbits underwent anterior cruciate ligament resection and were subsequently divided into four groups, composed of eleven subjects each. Group 1: rabbits received weekly intraarticular injections of saline over a period of four weeks; Group 2: the animals received three weekly intraarticular injections of native hyaluronic acid; Group 3: rabbits in this group received three weekly intraarticular injections of branched-chain hyaluronic acid; Group 4: two injections of betamethasone over a period of three weeks. A macroscopic study of the cartilage of the tibial plateaus was performed twelve weeks after surgery and changes in the joint surface were graded accordingly: Grade 0: smooth joint surface without relief changes; Grade 1: rough surface without any depression; Grade 2: similar to grade 1, but with depressions on the joint surface and Grade 3: subchondral bone exposure. Statistical analysis was performed with the use of Student's T test, chi-square test and analysis of variance (ANOVA), with a significance level of 5\%. Results: A statistical difference was found between the control group and the three study groups (2, 3 and 4) in both the development and severity of arthrosis. However, there was no difference in outcome among the three different drugs. Conclusion: A similar degree of attenuation of the osteoarthrosis process in rabbits' knees was found in all three study groups (low-molecular-weight glycosaminoglycans, high-molecular-weight glycosaminoglycans and betamethasone) when compared to placebo.

Keywords - Hyaluronic acid. Anterior cruciate ligament; Osteoarthritis; Knee; Rabbits

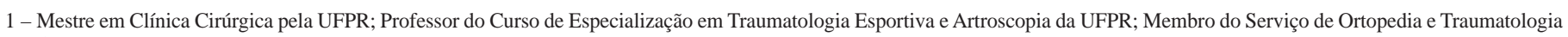
da UFPR.

2 - Médico Ortopedista; Aluno de Pós-graduação Universidade Federal do Paraná (SOT-UFPR).

3 - Médico Ortopedista; Aluno de Pós-graduação Universidade Federal do Paraná (SOT-UFPR).

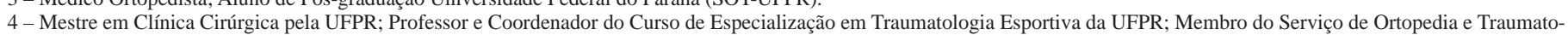
logia da UFPR; Membro Titular da SBOT.

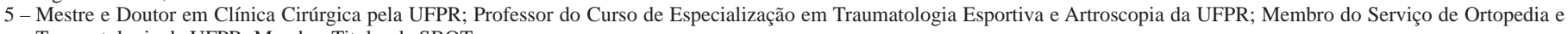
Traumatologia da UFPR; Membro Titular da SBOT.

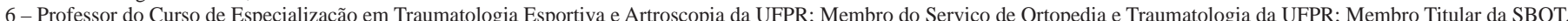

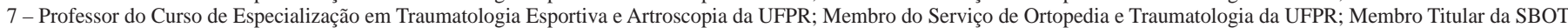

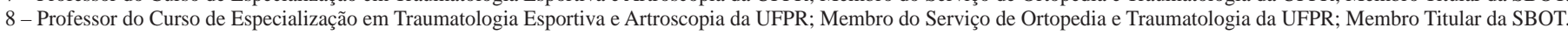

Trabalho realizado no Serviço de Ortopedia e Traumatologia da Universidade Federal do Paraná (SOT-UFPR) - Serviço do Prof. Dr. Luiz Antonio M. Da Cunha.

Correspondência: - Rua Padre Dehon 1119, ap. 410, V. Hauer - Curitiba, PR. E-mail: albano_mauro@yahoo.com.br 


\section{INTRODUÇÃO}

Osteoartrose é a enfermidade articular mais frequente da população senil, com prevalência superior a $10 \%$ após os 50 anos de idade, podendo acarretar importantes limitações funcionais ${ }^{(1,2)}$. Dentre as várias modalidades de tratamento disponíveis atualmente, a terapia com injeções intra-articulares de ácido hialurônico (AH) tem demonstrado efeito benéfico no controle dos sintomas da gonartrose ${ }^{(3)}$.

$\mathrm{O}$ AH, um polissacarídeo da família dos glicosaminoglicanos, contribui para a homeostase da articulação normal e apresenta-se em menor concentração e com peso molecular diminuído no líquido sinovial, nas articulações com osteoartrose ${ }^{(4-6)}$. O AH administrado na forma de injeções intra-articulares pode potencializar os efeitos regenerativos do $\mathrm{AH}$ endógeno sobre a cartilagem articular, restituindo a viscoelasticidade do líquido sinovial, contribuindo na síntese de AH pelos sinoviócitos, prevenindo a degradação de proteoglicanos e fibras de colágeno da matriz extracelular. Estimula o metabolismo e previne a apoptose dos condrócitos, inibe a degradação condral e as respostas inflamatórias articulares ${ }^{(6)}$. Atribui-se a estes efeitos da terapia com $\mathrm{AH}$ não só a sua capacidade em amenizar os sintomas relacionados à osteoartrose, mas também sua interferência na progressão da degeneração $\operatorname{articular}^{(4,5)}$.

Outra opção terapêutica no manejo da gonartrose são as injeções intra-articulares de corticosteroides. Estudos clínicos demonstraram resultados favoráveis em relação ao alívio de dor e melhora funcional, após injeções intra-articulares de fosfato dissódico de betametasona/acetato de betametasona ou metilprednisolona ${ }^{(7,8)}$. A administração intra-articular de corticosteroides em baixas a moderadas doses pode reduzir a progressão da erosão da cartilagem e a formação de osteófitos ${ }^{(9)}$. Além de suprimir a produção de metaloproteinases, implicadas na gênese da osteoartrose, é capaz de prevenir, parcialmente, a destruição de células e tecidos da matriz cartilaginosa por radicais livres de oxigênio ${ }^{(10,11)}$.

Considerando a abrangência e as implicações da osteoartrose de joelho, compreende-se atualmente a importância do diagnóstico e tratamento em suas fases iniciais, de maneira a minimizar suas consequências ${ }^{(12)}$. Por não haver até o presente momento intervenções que sejam capazes de inibir sua evolução, sobressaem as opções que permitem a redução da sua progressão. Injeções intra-articulares de diferentes tipos de ácido hialurônico e de corticosteroides poderiam ser utilizadas com esta finalidade.

Para avaliar os efeitos destas substâncias na gonartrose, propôs-se nesta pesquisa a utilização de um modelo experimental de osteoartrose que se assemelha àquela observada na espécie humana. A secção do ligamento cruzado anterior do joelho de coelhos mimetiza as alterações morfológicas e bioquímicas observada na osteoartrose humana, o que permite a reprodução acurada dos resultados obtidos ${ }^{(13-14)}$.

Objetivou-se neste trabalho avaliar o efeito das injeções intra-articulares do ácido hialurônico nativo (Polireumin ${ }^{\circledR}$, TRB Pharma, São Paulo, Brasil), do ácido hialurônico com cadeias ramificadas (Synvisc $^{\circledR}$, Novartis, São Paulo, Brasil) e do corticosteroide betametasona (Diprospan ${ }^{\circledR}$, Shering-Plough, São Paulo, Brasil) separadamente, e comparativamente entre si, na osteoartrose induzida pela secção do ligamento cruzado anterior (LCA) dos joelhos de coelhos.

\section{MÉTODOS}

Foram utilizados 44 coelhos da raça Califórnia, machos, que permaneceram alojados antes e durante a realização dos procedimentos, em Biotério, em gaiolas, com dois animais em cada uma, com alimentação padronizada e livre acesso à água. Foram mantidos em controle de luz (ciclo claro-escuro de 12 horas), com temperatura $\left(22 \pm 1^{\circ} \mathrm{C}\right)$, umidade e nível de ruído mantidos estáveis, até atingirem o peso médio de (3,5kg).

Todos os animais inicialmente foram submetidos à ressecção do LCA.

O procedimento cirúrgico consistiu em anestesia pré-operatoriamente com $10 \mathrm{mg} / \mathrm{kg}$ de cloridrato de cetamin (Dopalen ${ }^{\circledR}$ ) e 50mg/kg de cloridrato de xilasina $\left(\right.$ Anasedan $\left.{ }^{\circledR}\right)$, administradas na mesma seringa, de forma intramuscular (IM), no ventre dos músculos semimembranáceo e semitendíneo do membro posterior direito. Na mesma ocasião foi administrada injeção de 14.400UI de penicilinas e $6 \mathrm{mg}$ de estreptomicinas (Pentabiótico Veterinário Reforçado ${ }^{\circledR}$ - Eurofarma) como antibioticoprofilaxia, e Flunamine ${ }^{\circledR}$ (Bayer), na dose de 2,2mg/kg, por via IM, para analgesia pós-operatória. 
O joelho direito foi submetido à tricotomia e à antissepsia com polivinilpirrolidona (Povidini ${ }^{\circledR}$ ). Após a aplicação de campos estéreis, foi realizada incisão parapatelar medial na pele e tecido subcutâneo, seguida de capsulotomia e luxação lateral da patela.

O joelho foi então posicionado em flexão máxima, o que permitiu a visualização do ligamento cruzado anterior. Este foi seccionado com lâmina de bisturi $\mathrm{n}^{\circ}$ 15, a articulação foi irrigada com solução salina isotônica e procedeu-se à capsulorrafia e sutura da pele com mononáilon 4.0 (Figura 1).

Os coelhos permaneceram alojados nas suas respectivas gaiolas após o ato operatório, sem restrição de apoio aos membros operados. Foram distribuídos randomicamente em quatro grupos com 11 animais cada um. Decorridas três semanas do procedimento cirúrgico iniciaram-se as respectivas injeções intra-articulares.

Grupo 1: controle, injeções com solução salina isotônica, com intervalo de uma semana entre cada uma, por três semanas.

Grupo 2: três infiltrações, com intervalo de uma semana entre cada uma, com ácido hialurônico nativo (Polireumin ${ }^{\circledR}$ ).

Grupo 3: três infiltrações, com intervalo de uma semana entre cada uma, com ácido hialurônico ramificado (Synvisc ${ }^{\circledR}$ ).

Grupo 4: duas infiltrações, com intervalo de três semanas entre cada uma, com betametasona $0,25 \mathrm{mg}$ como dipropionato e $0,1 \mathrm{mg}$ como fosfato (Diprospan ${ }^{\circledR}$ ).
Um coelho do grupo 2 e outro do grupo 3 evoluíram com infecção do sítio operatório com extensão articular e foram excluídos da pesquisa.

Foram mortos após 12 semanas do ato cirúrgico. Para isso foram previamente anestesiados como descrito anteriormente e submetidos a injeção intracardíaca de tiopental $(5 \mathrm{ml})$ e cloreto de potássio $(10 \mathrm{ml})$.

Os platôs tibiais foram ressecados assepticamente, imersos em frasco contendo formol a 10\%, para posterior avaliação da cartilagem articular por exame macroscópico.

As alterações na superfície articular foram graduadas pela macroscopia da seguinte maneira: grau 0 - superfície articular lisa, brilhante, sem alterações de relevo; grau 1 - superfície rugosa, com perda do brilho, sem depressões; grau 2 - semelhante ao grau 1, com depressões na superfície; grau 3 - exposição do osso subcondral (Figura 2). Os platôs tibiais lateral e medial foram avaliados separadamente em cada grupo.

A análise estatística consistiu na utilização do teste $t$ de Student para determinar se houve diferença entre os diferentes grupos em relação aos graus de osteoartrose, avaliada pela macroscopia. Para determinar se houve diferença interna nos grupos quanto ao grau de osteoartrose nos compartimentos lateral e medial foi realizado o teste do Qui-quadrado, e para verificar diferença entre os graus de osteoartrose, independentemente da droga utilizada, utilizou-se a análise de variância (Anova).
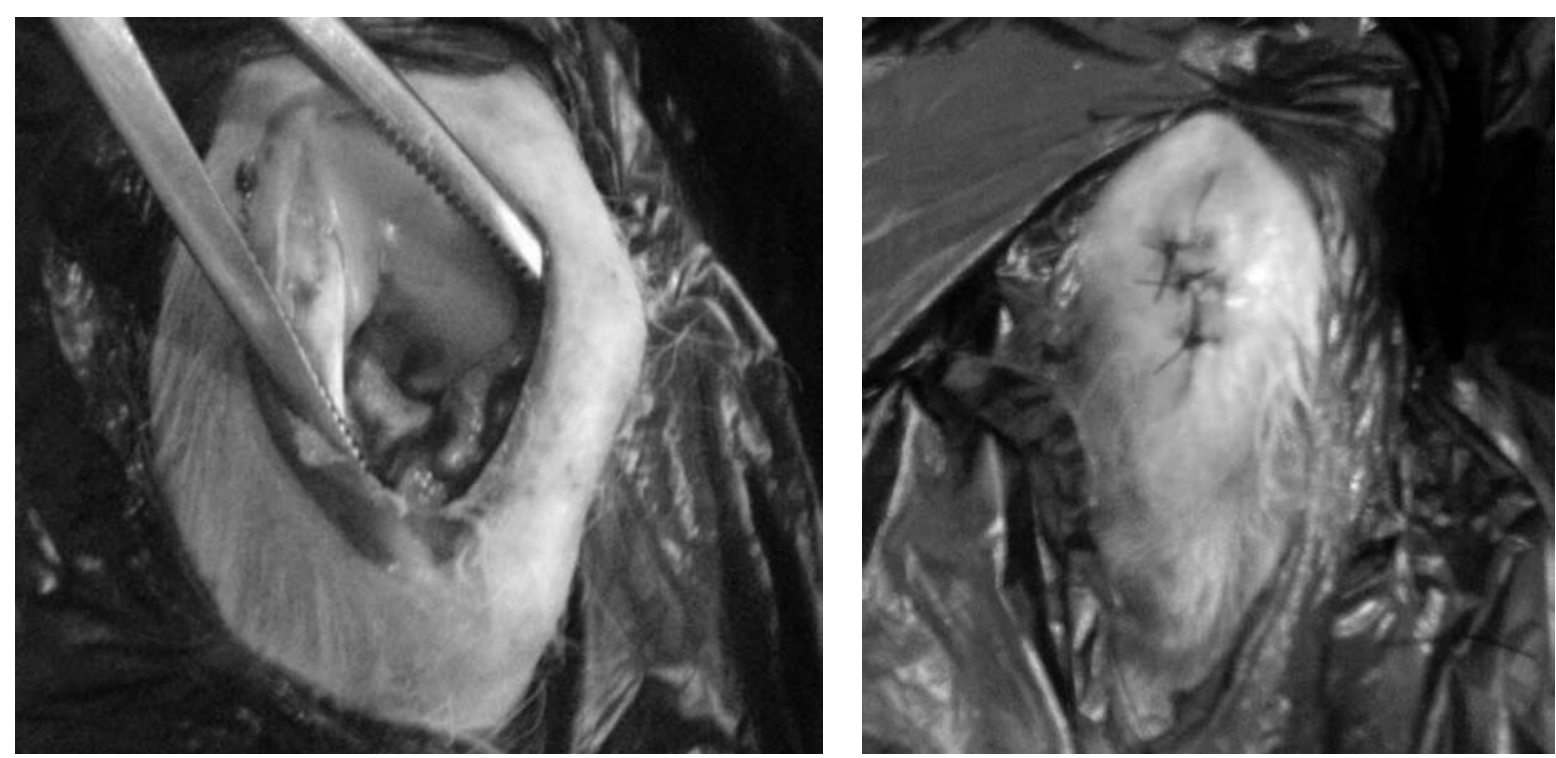

Figura 1 - Fotografias demonstrando a exposição cirúrgica do ligamento cruzado anterior e o fechamento da pele após a ressecção 


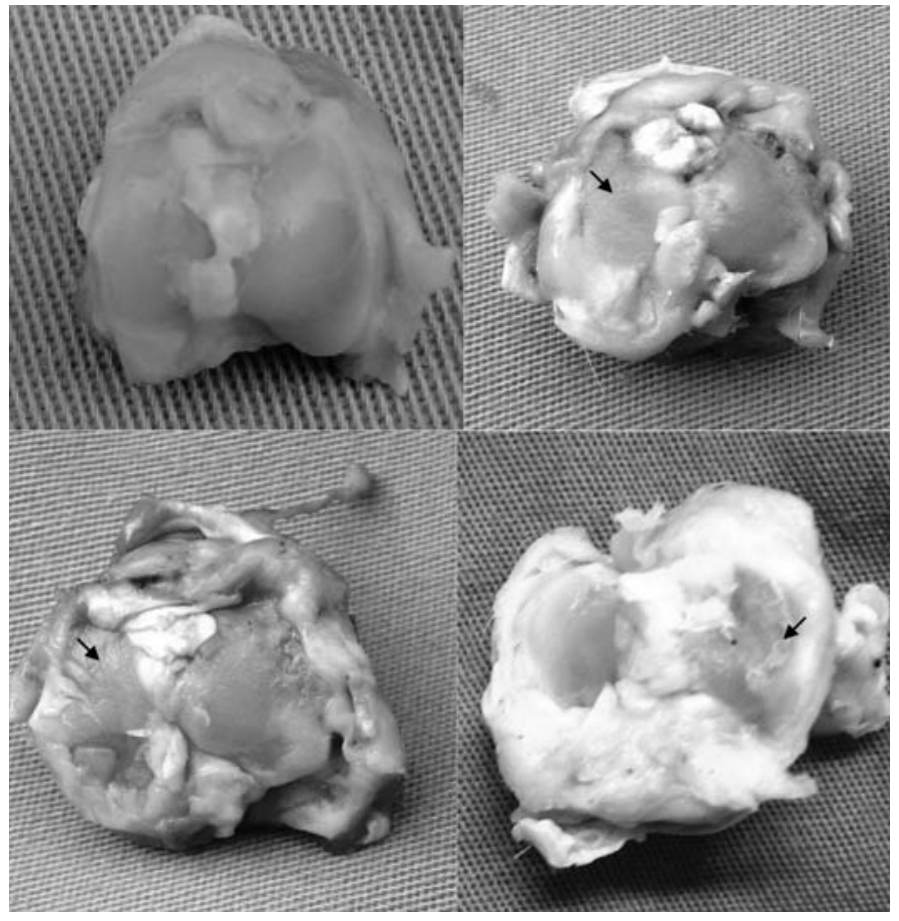

Figura 2 - Classificação macroscópica dos platôs tibiais A) grau 0; B) grau 1 (seta: perda do brilho, rugosidade); C) grau 2 (seta: depressão superficial); D) grau 3 (seta: exposição do osso subcondral)

\section{RESULTADOS}

A osteoartrose induzida pela ressecção do ligamento cruzado anterior, no grupo placebo, ocorreu nos dois compartimentos em todos os joelhos. Nos grupos 2, 3 e 4 ocorreram em 40\%, 65\% e 45\% dos compartimentos, respectivamente (Tabela 1 e Figura 3).

O nível de significância utilizado para todas as análises foi de $5 \%$.

Utilizando o teste $t$ de Student para comparar as diferentes drogas e o placebo, quanto ao desenvolvi-

Tabela 1 - Frequência dos diferentes graus de osteoartrose nos compartimentos medial e lateral dos grupos de estudo

\begin{tabular}{c|c|c|c|c|c|c|c|c}
\hline $\begin{array}{c}\text { Graus } \\
\text { da } \\
\text { doença }\end{array}$ & placebo & $\%$ & polireumin & $\%$ & synvisc & $\%$ & corticoide & $\%$ \\
\hline G0 & 0 & 0,00 & 12 & 60,00 & 7 & 35,00 & 12 & 54,54 \\
\hline G1 & 13 & 59,09 & 1 & 5,00 & 7 & 35,00 & 7 & 31,82 \\
\hline G2 & 5 & 22,73 & 7 & 35,00 & 6 & 30,00 & 3 & 13,64 \\
\hline G3 & 4 & 18,18 & 0 & 0,00 & 0 & 0,00 & 0 & 0,00 \\
\hline Total & 22 & 100,00 & 20 & 100,00 & 20 & 100,00 & 22 & 100,00 \\
\hline
\end{tabular}

\section{TOTAL DE J OELHOS POR TIPO DE DROGA E GRAU DA DOENÇA}

$\square \mathrm{G} 0 \quad \square \mathrm{G} 1 \quad \square \mathrm{G} 2 \quad \mathrm{G} 3$

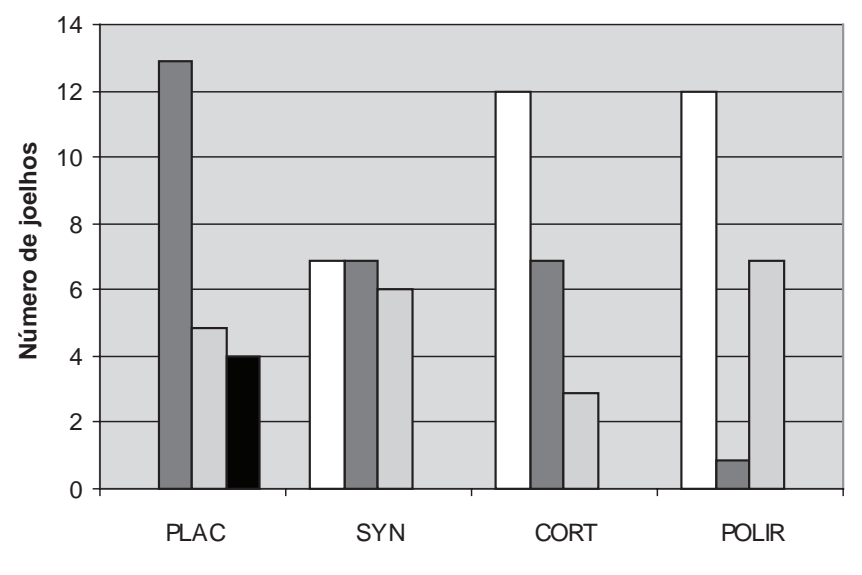

Figura 3 - Ocorrência de osteoartrose conforme o grau nos planaltos tibiais nos diferentes grupos

mento de osteoartrose, observou-se diferença estatística entre os grupos 2, 3 e 4 e o grupo controle (Tabela 2), porém não houve diferença entre os grupos das drogas estudadas considerando $\mathrm{p}<0,05$.

Tabela 2 - Teste $t$ de Student

\begin{tabular}{c|c}
\hline Drogas & T calculado \\
\hline synvisc/ corticoide & 0,8232 \\
\hline synvisc/ polireumin & 1,0000 \\
\hline corticoide/ polireumin & 0,8790 \\
\hline
\end{tabular}

O valor de $t$ crítico para todas as comparações é de 2,7764

Nível de significância de $5 \%$

Por meio da Anova, constatou-se existir diferença entre os diferentes graus de osteoartrose, independente da droga utilizada (Tabela 3).

Tabela 3 - Teste de variância (Anova)

\begin{tabular}{c|c|c}
\hline Fonte de variação & F & P valor \\
\hline Graus da doença & 6,5890 & 0,025 \\
\hline
\end{tabular}

Ao se comparar dentro de cada grupo o grau de osteoartrose entre os compartimentos medial e lateral, constatou-se não haver diferença significativa em nenhum dos grupos de estudo, pelo teste do Qui-quadrado (Tabela 4). 
Tabela 4 - Teste do Qui-quadrado

\begin{tabular}{c|c}
\hline Drogas & calculado \\
\hline placebo & 0,8707 \\
\hline synvisc & 0,0631 \\
\hline corticoide & 0,1066 \\
\hline polireumin & 0,0040 \\
\hline Valor do Qui-quadrado tabelado com 0,05 de significância e 1 grau de liberdade é igual a
\end{tabular}

3,84

\section{DISCUSSÃO}

Neste experimento, a cartilagem articular dos platôs tibiais medial e lateral dos joelhos de coelhos submetidos à secção do ligamento cruzado anterior e à infiltrações de hialuronatos, corticosteroide e solução salina isotônica no grupo controle foi avaliada por macroscopia. A definição dos diferentes graus de osteoartrose baseou-se na observação do aspecto da superfície da cartilagem articular, variando de normal (grau 0) até a destruição completa da cartilagem e exposição do osso subcondral (grau 3). Com base nesta classificação observou-se, em todos os joelhos infiltrados com solução salina isotônica, algum grau de lesão condral e degeneração articular grave (grau 3) em $18 \%$. Nos demais grupos houve casos que não evoluíram para artrose e no máximo se observaram alterações do relevo da superfície articular (Tabela 1 e Figura 3).

Os resultados demonstraram haver diferença estatística, quanto à prevenção de lesão condral, entre os grupos que receberam injeções articulares de diferentes tipos de ácido hialurônico e de corticosteroide, em relação ao grupo placebo (Tabela 2). Não se observou preponderância de lesão articular entre os compartimentos tibiais lateral e medial em nenhum dos grupos (Tabela 4). Da mesma forma que outros trabalhos citados na literatura este estudo demonstrou efeito condroprotetor após as injeções intra-articulares dos glicosaminoglicanos de baixo e alto peso molecular, e do corticosteroide ${ }^{(5,15-18)}$. Está em concordância com o trabalho de Karakurum et $a l^{(8)}$ que compararam as injeções intra-articulares de predinisolona com as de ácido hialurônico, em um modelo de osteoartrose experimental em coelhos e observaram prevenção da degeneração articular na mesma extensão por ambas as drogas administradas isoladamente e um efeito potencializado quando administradas em conjunto.
Para as infiltrações com corticosteroides foram utilizadas doses proporcionais às utilizadas no tratamento humano, evitando-se desta forma o agravamento das lesões condrais por excesso de medicamento. O efeito condroprotetor observado em diferentes estudos ocorreu com o uso de corticosteroides em baixas doses. O intervalo de três semanas entre as aplicações do corticosteroide foi adotado pelo fato de que os corticosteroides menos solúveis podem permanecer no interior da articulação por este período ${ }^{(19)}$.

Yoshimi et al $^{(4)}$ desenvolveram o modelo de osteoartrose pela secção do ligamento cruzado anterior em joelho de coelhos. Comparativamente a outros modelos, como a meniscectomia medial parcial em coelhos e a secção do ligamento cruzado anterior em cães e ratos é o que mais se aproxima do processo de degeneração articular em humanos ${ }^{(20-25)}$. Os artigos publicados na literatura que dele se utilizaram para avaliar o efeito condroprotetor dos glicosaminoglicanos e dos corticosteroides demonstraram, na grande maioria dos casos, a evolução dos grupos controles para $\operatorname{artros}^{(4,5,8-10,14,15,24-26)}$. Estudos clínicos prospectivos e randomizados atuais, contrariamente aos estudos experimentais, não comprovam o efeito condroprotetor dos diferentes tipos de ácido hialurônico e corticosteroides. O que se apresenta como evidência são a melhora nos níveis de dor e na função para atividades de vida diária para os pacientes que fizeram uso destas medicações, no curto prazo ${ }^{(27-38)}$. São necessários estudos controlados com maior período de acompanhamento para se esclarecer o papel destas drogas na prevenção da degeneração da cartilagem articular.

A continuidade desta pesquisa pretende avaliar, pela histologia e pela quantificação histomorfométrica dos glicosaminoglicanos da matriz extracelular, a influência das mesmas substâncias no modelo de osteoartrose aqui utilizado.

\section{CONCLUSÃO}

No presente estudo conclui-se existir atenuação da evolução do processo de osteoartrose, na mesma magnitude, com a utilização de injeções intra-articulares dos glicosaminoglicanos de baixo peso molecular, de alto peso molecular, assim como do corticosteroide composto por dipropionato betametasona e fosfato dissódico de betametasona, em joelhos de coelhos. 


\section{REFERÊNCIAS}

1. Wang CT, Lin J, Chang CJ, Lin YT, Hou SM. Therapeutic effects of hyaluronic acid on osteoarthritis of the knee. J Bone Joint Surg Am. 2004;86(3):538-45.

2. Bedson J, Jordan K, Croft P. The prevalence and history of knee osteoarthritis in general practice: a case-control study. Fam Pract. 2005;22(1):103-8.

3. Altman RD, Moskovitz R. Intraarticular sodium hyaluronate in the treatment of patients with osteoarthritis of the knee: a randomized clinical trial. J Rheumatol. 1998;25(11):2203-12.

4. Yoshimi T, Kikuchi T, Obara T, Yamaguchi T, Sakakibara Y, Itoh H, et al. Effects of high-molecular-weight sodium hyaluronate on experimental osteoarthrosis induced by the resection of rabbit anterior cruciate ligament. Clin Orthop Relat Res. 1994;(298):296-304.

5. Hulmes DJ, Marsden ME, Strachan RK, Harvey RE, McInnes N, Gardner DL. Intra-articular hyaluronate in experimental rabbit osteoarthritis can prevent changes in cartilage proteoglycan content. Osteoarthritis Cart. 2004;12(3):232-8.

6. Schiavinato A, Finesso M, Cortivo R, Abatangelo G. Comparison of the effects of intra-articular injections of Hyaluronan and its chemically cross-linked derivative. Clin Exp Rheumatol. 2002;20(4):445-54.

7. Leopold SS, Redd BB, Warme WJ, Wehrle PA, Pettis PD, Shott S. Corticosteroid compared with hyaluronic acid injections for the treatment of osteoarthritis of the knee. J Bone Joint Surg Am. 2003;85(7):1197-203.

8. Karakurum G, Karakok M, Tarakcioglu M, Koger NE, Kocabas R, Bagci C. Comparative effect of intra-articular administration of hyaluronan and/or cortisone with evaluation of malondialdehyde on degenerative osteoarthritis of the rabbit's knee. Tohoku J Exp Med. 2003;199(3):127-34.

9. Pelletier JP, Mineau F, Raynauld JP, Woessner JF, Gunia-Smith Z, Pelletier JM. Intraarticular injections with methylprednisolone acetate reduce osteoarthritic lesions in parallel with condrocyte stromelysin synthesis in experimental osteoarthritis. Arthritis Rheum. 1994;37(3):414-23.

10. Pelletier JP, Cloutier JM, Martel-Pelletier J. In vitro effects of tiaprofenic acid, sodium sodium salicylate and hydrocortisone on the proteoglycan metabolism of human osteoarthritic cartilage. Rheumatol. 1989;16(5):646-55.

11. Cortivo R, Brun P, Cardarelli L, O'regan M, Radice M, Abatangelo G. Antioxidant effects of hyaluronan and its alpha-methyl-prednisolone derivative in chondrocyte and cartilage cultures. Semin Arthritis Rheum. 1996;26(1):492-501.

12. Cooper C, Snow S, McAlindon TE, Kellingray S, Stuart B, Coggon D, et al. Risk factors for the incidence and progression of radiographic knee osteoarthritis. Arthritis Rheum. 2000;43(5):995-1000.

13. Moskowitz RW. Experimental models of osteoarthritis. In: Moskovitz RW, Altman RD, Buckwalter JA, Goldberg VM, Hochberg MC. Osteoarthritis: diagnosis and medical/surgical management. 2th. Philadelphia: Saunders; 1992. p. 213-52.

14. Sah RL, Yang AS, Chen AC, Hant JJ, Halili RB, Yoshioka M, et al. Physical properties of rabbit articular cartilage after transaction of the anterior cruciate ligament. J Orthop Res. 1997;15(2):197-203.

15. Kobayashi K, Amiel M, Harwood FL, Healey RM, Sonoda M, Moriya H, et al. The long-term effects of hyaluronan during development of osteoarthritis following partial meniscectomy in a rabbit model. Osteoarthritis Cart. 2000;8(5):359-65.

16. Butler M, Colombo C, Hickman L, O'Byrne E, Steele R, Steinetz B, et al. A new model of osteoarthritis in rabbits. III. Evaluation of anti-osteoarthritis effects of selected drugs administered intraarticularly. Arthritis Rheum. 1983;26(11):1380-6.

17. Pelletier JP, Mineau F, Raynauld JP, Woessner JF Jr, Gunja-Smith Z, MartelPelletier J. Intraarticular injection with methylprednisolone acetate reduces osteoarthritis lesions at the same time as chondrocyte stromelysin synthesis in experimental osteoarthritis. Arthritis Rheum. 1994;37(3):414-23.

18. Pelletier JP, DiBattista JA, Raynauld JP, Wilhelm S, Martel-Pelletier J. The in vivo effects of intraarticular corticosteroid injections on cartilage lesions, stromelysin, interleukin-1, and oncogene protein synthesis in experimental osteoarthritis. Lab Invest. 1995;72(5):578-86.
19. Caldwell, JR. Intra-articular corticosteroids: guide to selection and indications for use. Drugs 1996;52(4):507-14.

20. Moskowitz RW, Davis W, Sammarco J, Martens M, Baker J, Mayor M, et al. Experimentally induced degenerative joint lesions following partial meniscectomy in the rabbit. Arthritis Rheum. 1973;16(3):397-405.

21. Moskowvitz RW, Howell DS, Goldberg VM, Muniz O, Pita JC. Cartilage proteoglycan alterations in a experimentally induced model of rabbit osteoarthritis. Arthritis Rheum. 1979;22(2):155-63.

22. Pond MJ, Nuki G. Experimentally-induced osteoarthritis in the dog. Ann Rheum Dis. 1973;32(4):387-8.

23. Stoop R, Buma $P$, van der Kraan PM, Hollander AP, Billinghurst RC, Meijers TH, et al. Type II collagen degradation in articular cartilage fibrillation after anterior cruciate ligament transaction in rats. Osteoarthritis Cart. 2001;9(4):308-15.

24. Amiel D, Toyoguchi T, Kobayashi K, Bowden K, Amiel M, Healey RM. Long-term effect of sodium hyaluronate $\left(\mathrm{Hyalgan}^{\circledR}\right)$ on osteoarthritis progression in a rabbit model. Osteoarthritis Cart. 2003;11(9):636-43.

25. Yoshioka M, Coutts RD, Amiel D, Hacker SA. Characterization of a model of osteoarthritis in the rabbit knee. Osteoarthritis Cart. 1996;4(2):87-98.

26. Chang DG, Iverson EP, Schinagl RM, Sonoda M Amiel D. Coutts RD, et al. Quantitation and localization of cartilage degeneration following the induction of osteoarthritis in the rabbit knee. Osteoarthritis Cart. 1997;5(5):357-72.

27. Divine JG, Zazulak BT, Hewett TE. Viscosupplementation for knee osteoarthritis. A systematic review. Clin Orthop Relat Res. 2006;(455):113-22.

28. Kemper F, Gebhardt U, Meng T, Murray C. Tolerability and short-term effectiveness of hylan G-F 20 in 4253 patients with osteoarthritis of the knee in clinical practice. Current Med Res Opin. 2005;21(8):1261-9.

29. Waddell DD, Cefalu CA, Bricker DC. A second course of hylan G-F 20 for the treatment of osteoarthritic knee pain: 12-month patient follow-up. J Knee Surg. 2005;18(1):7-15

30. Waddell D, Bricker DC. Clinical experience with the effectiveness and tolerability of hylan G-F 20 in 1047 patients with osteoarthritis of the knee. J Knee Surg. 2006;19(1):19-27.

31. Raynauld JP, Goldsmith CH, Bellamy N, Torrance GW, Polisson R, Belovich D, et al. Effectiveness and safety of repeat courses of hylan G-F 20 in the patients with knee osteoarthritis. Osteoarthritis Cart. 2005;13(2):111-9.

32. Pyne D, Ioannou Y, Mootoo R, Bhanji A. Intra-articular steroids in knee osteoarthritis: a comparative study of triancinolone hexacetonide and methylprednisolone acetate. Clin Rheumatol. 2004;23(2):116-20.

33. Leopold SS, Warne WJ, Pettis PD, Shott S. Increased frequency of acute local reaction to intra-articular hylan GF 20 (Synvisc) in patients receiving more than one course of treatment. J Bone J Surg Am. 2002;84(9):1619-23.

34. Pagnano M, Westrich G. Succesful nonoperative management of chronic osteoarthritis pain of the knee: safety and efficacy of retreatment with intra-articular hyaluronans. Osteoarthritis Cart. 2005;13(9):751-61.

35. Marino A, Wadell D, Kolomytkin O, Pruett S, Sadasivan K, Albright J. Assessment of immunologic mechanisms for flare reactions to Synvisc. Clin Orthop Relat Res. 2006;(442):187-94.

36. Bellamy N, Campbell J, Robinson V, Gee T, Bourne R, Wells G. Viscosupplementation for osteoarthritis of the knee. Cochrane Database Syst Rev. 2006:CD005321.

37. Kotevoglu N, Ivibozkurt PC, Hiz O, Toktas $\mathrm{H}$, Kuran $\mathrm{B}$. A prospective randomised controlled clinical trial comparing the efficacy of different molecular weight hyaluronan solutions in the treatment of knee osteoarthritis. Rheumatol Int. 2006;26(4):325-30

38. Bellamy N, Campbell J, Robinson V, Gee T, Bourne R, Wells G. Intraarticular corticosteroid for treatment of osteoarthritis of the knee. Cochrane Database Syst Rev. 2005:CD005328 